\title{
A Composition-Transferable Machine Learning Potential for LiCl-KCl Molten Salts Validated by HEXRD
}

\author{
Jicheng Guo \\ Chemical and Fuel Cycle Technologies Division, \\ Argonne National Laboratory, Lemont, IL 60439, USA \\ Logan Ward and Yadu Babuji \\ Data Science and Learning Division, Argonne National Laboratory, Lemont, IL 60439, USA \\ Nathaniel Hoyt and Mark Williamson \\ Chemical and Fuel Cycle Technologies Division, Argonne National Laboratory, Lemont, IL 60439, USA \\ Ian Foster \\ Data Science and Learning Division, Argonne National Laboratory, Lemont, IL 60439, USA \\ Nicholas Jackson \\ Department of Chemistry, University of Illinois, Urbana-Champaign, Urbana, IL 61801, USA \\ Chris Benmore \\ X-ray Science Division, Argonne National Laboratory, Lemont, IL 60439, USA \\ Ganesh Sivaraman* \\ Data Science and Learning Division, \\ Argonne National Laboratory, Lemont, IL 60439, USA
}

(Dated: February 2, 2022)

\begin{abstract}
Unraveling the liquid structure of multi-component molten salts is challenging due to the difficulty in conducting and interpreting high temperature diffraction experiments. Motivated by this challenge, we developed composition-transferable Gaussian Approximation Potentials (GAP) for molten LiCl-KCl. A DFT-SCAN accurate GAP is active learned from only 1100 training configurations drawn from 10 unique mixture compositions enriched with metadynamics. The GAP-computed structures show strong agreement across HEXRD experiments, including for a eutectic not explicitly included in model training, thereby opening the possibility for composition discovery.
\end{abstract}

Molten salts (MS) are a class of high temperature ionic liquids relevant to liquid metal batteries, concentrated solar power systems, and molten salt reactors [1-5]. Critical to technological applications of MS are the eutectic mixtures of alkali/alkali-earth halides, whose melting temperatures can be lowered by tuning the mixture composition $[6,7]$. Tuning the thermophysical properties of multi-component salts requires a precise atomistic understanding of the liquid structure; challenges for this task include (a) the difficulty of in situ experimental measurements, due to the extreme reactivity of molten salts, (b) the lack of a priori knowledge regarding optimal eutectic compositions for different MS chemistries [8], and (c) the inability of conventional molecular modeling approaches to capture the complex intermolecular interactions and spatiotemporal scales characteristic of molten salts [9$14]$.

Machine learning (ML) has enabled [15-18] a new generation of low-cost interatomic potentials (IP) that provide access to quantum mechanically accurate manybody potential energy surfaces for condensed phases [19-
28]. These ML-IP can drive mesoscopic simulations of atomic processes with ab initio accuracy, bypassing the length scale limitations imposed by traditional $a b$ initio methods. Recent efforts have begun applying these methods to single and multi-component MS [29-33]. However, current ML-IP for MS are generally fit to a small number of mixture compositions (including a priori known eutectic compositions) and lack transferability to arbitrary or unknown mixture compositions. This lack of transferability inhibits eutectic discovery for arbitrary MS chemistries, and limits existing ML-IP to simple interpolative efforts between known MS compositions. Thus there is an urgent need for ML-IP of MS exhibiting multi-component, compositional transferability across the space of possible anion and cation combinations.

LiCl-KCl mixtures exhibit high potential for molten salt reactors, pyroprocessing and energy storage applications due to their preferable physiochemical properties such as low melting point, high solubility of fission products, and high heat capacity [34-36]. In this letter, 
we develop a LiCl-KCl ML-IP with broad compositional transferability, and apply it to ascertain the compositiondependent structure of $\mathrm{LiCl}-\mathrm{KCl}$ mixtures. The compositional transferability of this methodology opens the door for a priori prediction, by molecular modeling, of eutectic compositions for MS. Finally, we demonstrated the use of ML-IP to accurately compute thermal conductivity of the eutectic molten $\mathrm{LiCl}-\mathrm{KCl}$.

We have designed a combined experimental and modeling workflow for investigating multiple compositions of LiCl-KCl melts (Fig. 1). The modeling component of the workflow leads to the generation of a multi-composition ML-IP, specifically a Gaussian approximation potential (GAP) [19], for LiCl-KCl. The GAP model uses twobody squared exponential and many-body smooth overlap of atomic positions (SOAP) kernel functions [37, 38] to measure chemical similarity between local chemical neighborhoods. The short-range GAP model is a reasonable choice given that the goal is to model bulk liquid structure [29, 39]. However, explicit inclusion of longrange electrostatics is necessary for modeling scenarios requiring inclusion of nonisotropic chemical environments such as interfaces or electric field [40]. GAP modeling training utilizes atomic configurations drawn from a diverse set of melt compositions. Configurational sampling (Fig. 1a) was initialized with 10 unique compositions hand picked by the experimentalist, listed here as molar fractions of $\mathrm{KCl}(100 \%, 90 \%, 80 \%, 67 \%, 58 \%, 50 \%$, $33.3 \%, 30 \%, 20 \%$, and $10 \%$ ). Initial simulation densities are fixed to literature values [41]. At each composition, a small unit cell of 60-64 atoms is used to sample a range of melted atomic configurations by using the Born-MayerHuggins-Tosi-Fumi (BMHTF) rigid ion model (RIM) at an elevated temperature of $2100 \mathrm{~K}$. Configuration sampling was explicitly not performed for the middle of the composition map between $50 \%$ to $33.3 \% \mathrm{KCl}$ molar fraction. This conscious choice was made in order to not include the actual eutectic composition of 58.5-41.5 mol\% $\mathrm{LiCl}-\mathrm{KCl}$ as part of the training set. By doing so the ability of the generated GAP model to accurately predict compositions that are not explicitly included in the training data could be confirmed.

Configurational sampling for each composition uses approximately 20,000 melt configurations drawn at $2100 \mathrm{~K}$ and processed by using active learning (AL). The AL consists of an unsupervised clustering algorithm combined with Bayesian optimization for on-the-fly hyperparameter tuning of the GAP model. A detailed description of the AL procedure can be found in previous work [39, 42, 43]. Single point DFT calculations (Fig. 1b) are performed on the AL-extracted configurations by using the SCAN exchange correlation (XC) functional [44], which shows superior performance compared to generalized gradient approximation XC functionals [45]. Further details on the DFT calculations are reported in the supplementary material section B.

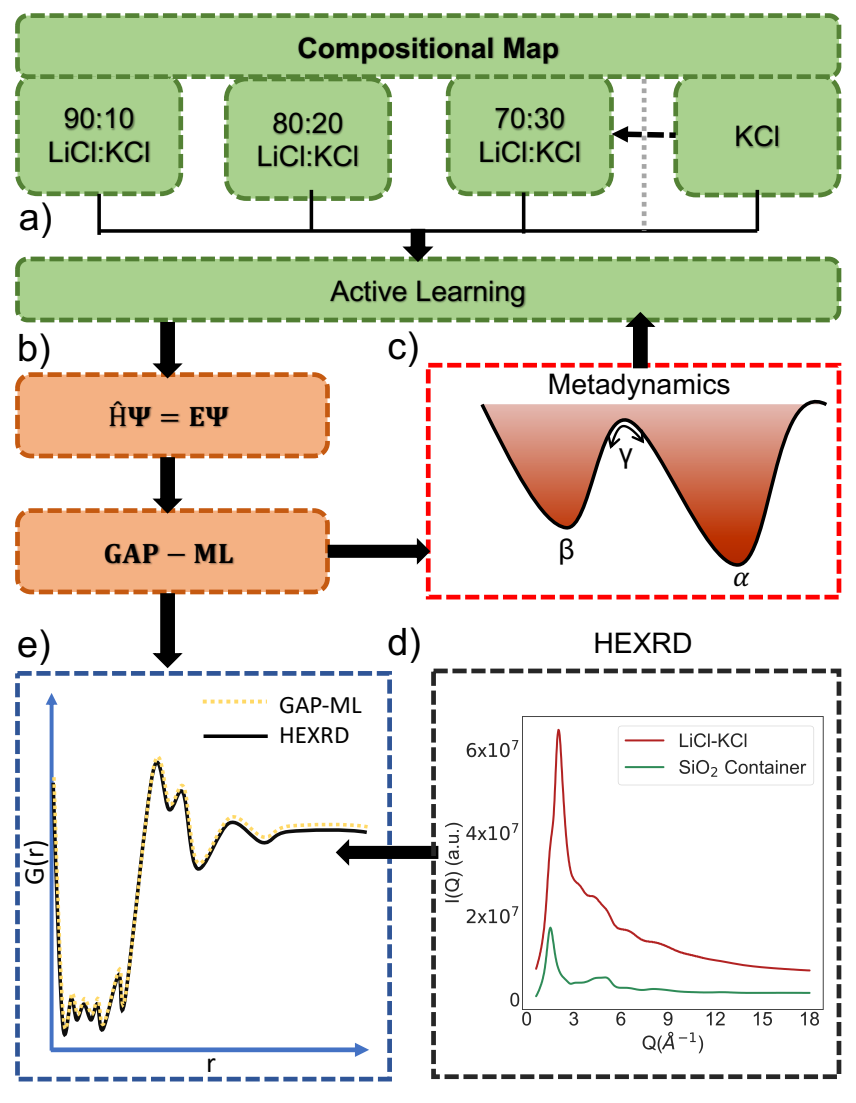

FIG. 1. Workflow for mapping and validating multicomposition LiCl-KCl melts. (a) Sample the configuration space for 10 unique compositions of pure $\mathrm{KCl}$ to $10 \mathrm{~mol} \% \mathrm{KCl}$ mixture with $\mathrm{LiCl}$. Each composition is melted by using the rigid ion model at $2100 \mathrm{~K}$. Clustering-based AL enables down selection. Ensemble simulations and AL are orchestrated by using the Colmena framework [46]. (b) Perform single point DFT for the AL samples and fit the GAP-ML model. (c) Enrich the configuration space by using metadynamics [47] on the GAP-ML based MD. (d) High energy X-ray diffraction experiments. Illustration of X-ray intensity measurement. (e) Perform rigorous validation of GAP-ML driven MD simulation by using the high energy X-ray pair distribution functions (PDFs).

An initial GAP model is fitted to the AL-extracted configurations for the 10 compositions (Fig. 1b), with "labels" computed by using DFT-SCAN calculations. Since all training configurations are drawn from equilibrium MD simulations, there is no guarantee of configurational or compositional transferability for the GAP model. To circumvent the limitations of Boltzmann sampling, strategies such as random structure search and enhanced sampling have been applied to enrich training databases [48, 49]. Here, we employ metadynamics to construct a history-dependent repulsive potential as a function of a set of collective variables $(\mathrm{CVs})$, providing access to a large configuration space of ion pair 
coordination environments that would be unexplored by simple equilibrium MD [47, 50]. We hypothesize that the increased configurational diversity at each training composition should manifest in improved compositional transferability across the $\mathrm{LiCl}-\mathrm{KCl}$ composition range.

Enhanced sampling is performed by using the welltempered variation of metadynamics for an equal fraction of $\mathrm{LiCl}$ and $\mathrm{KCl}$ (i.e., 50\%) [51]. A system size of 64 atoms near the melting temperature $(\sim 757 \mathrm{~K})$ is simulated by using the atomic pair coordination collective variable $(\mathrm{CV})$ parameterized by the first minimum of the pair distribution function (PDF). Further information on the metadynamics procedure is discussed in supplementary material section C. The evolution of the CV over $4 \mathrm{~ns}$ is shown in supplementary material section $\mathrm{C}$ Fig. 1. In equilibrium MD the sampled configuration will be dominated by $\sim 4$ and $\sim 7$ coordinated $\mathrm{Li}-\mathrm{Cl}$ and $\mathrm{K}-\mathrm{Cl}$, respectively (Table I). Metadynamics facilitates efficient exploration of out-of-equilibrium regions of configuration space. The configurations obtained from over $4 \mathrm{~ns}$ of metadynamics simulation were passed to $\mathrm{AL}$ and down-selected. It is observed that $\mathrm{AL}$ efficiently samples from near and far away from equilibrium regions of metadynamics configurations, thereby enriching the training database (supplementary material section C Fig. 1). The final GAP model is trained by using the metadynamics-enriched dataset. The details of the GAP training database and hyperparameters are discussed in the supplementary material section A Tables I, II. The entire GAP model is generated with only 1,127 training samples which is also the total number of single point DFT performed as a part of this letter, effectively bypassing expensive ab initio molecular dynamics. A single compute node benchmark with 64 atoms unit cell of 50-50 mol\% showed 15,000 $\times$ speedup of GAP-MD $(\sim 0.042 \mathrm{~s} / \mathrm{MD}$ time step) relative to DFT-SCAN $(\sim 618$ $\mathrm{s} / \mathrm{MD}$ time step).

High energy X-ray diffraction (HEXRD) experiments were performed to characterize the structure of molten LiCl-KCl mixtures in parallel with the modeling work (Fig. 1). For HEXRD experiments, LiCl-KCl mixture samples were prepared in an ultra-high purity Ar glovebox $\left(<1 \mathrm{ppm} \mathrm{O}_{2},<1 \mathrm{ppm} \mathrm{H}_{2} \mathrm{O}\right)$. Samples with the desired $\mathrm{LiCl}-\mathrm{KCl}$ ratio were melted in glassy carbon crucibles to create homogeneous mixtures, then the solidified mixtures were crushed and loaded into silica glass $\left(\mathrm{SiO}_{2}\right)$ ampules and sealed under vacuum for the experiments. HEXRD experiments were performed at beamline 6-ID-D of the Advanced Photon Source, Argonne National Laboratory. LiCl-KCl mixtures were heated to the desired temperatures at which diffraction data was recorded. The diffraction patterns of empty furnace and empty glass ampules were also recorded for background subtraction. The diffraction data of $\mathrm{LiCl}-\mathrm{KCl}$ with several compositions including 70-30 mol\%, 58.5-41.5 mol\% (eutectic composition) and 50-50 mol\% were recorded at multiple temperatures. Temperature dependence is studied by measuring the structures of $70-30 \mathrm{~mol} \%$ at $\sim 822 \mathrm{~K}, 797 \mathrm{~K}, 772 \mathrm{~K}$, and $747 \mathrm{~K}$. The $58.5-41.5 \mathrm{~mol} \%$ (eutectic composition) and 50-50 mol\% are measured near the melting temperatures of $\sim 645 \mathrm{~K}$ and $757 \mathrm{~K}$, respectively.

The final GAP model obtained from the workflow in Fig. 1 is used to perform simulated annealing with a system size over $\sim 1000$ atoms at each of the experimental compositions and temperatures (supplementary material section D Table III ) [52]. We performed GAP MD using the LAMMPS software package compiled with the QUIP pair style [53, 54]. The structure prediction with simulated annealing is performed in three steps: 1) Each simulation condition is initially thermalized at $1100 \mathrm{~K}$ in the (NVT) ensemble [55, 56], followed by volume relaxation in an isothermal-isobaric (NPT) ensemble with a pressure coupling of 1 bar [57-59], 2) the temperature is increased to $1700 \mathrm{~K}$ over $200 \mathrm{ps}$ in the NPT ensemble, and 3 ) the system is cooled from $1700 \mathrm{~K}$ to the target temperature over $200 \mathrm{ps}$ of NPT simulations. All heating and cooling MD simulations used a time step of $0.5 \mathrm{fs}$. At the target temperature the volume of the system is relaxed for over $2 \mathrm{~ns}$ with a time-step of $1.0 \mathrm{fs}$ and the last $1 \mathrm{~ns}$ is used for computing the structure. Further information on the number of atoms, starting densities, and densities estimated from GAP-MD for each composition and temperature are listed in supplementary material section D Table III (includes outside of experiment condition).

To determine the quality of the generated GAP model, the structures obtained from simulations are validated by comparison to HEXRD measurements. The structure factors and PDFs from experiments and simulations are shown in Fig. 2. Note that such a comparison at temperatures just above the melting points has not been previously reported. The simulated PDF (G(r)) and structure factors (S(q)) exhibit excellent agreement with those obtained from HEXRD across all examined compositions. Specifically, GAP accurately predicts the structure of the eutectic (Fig. 2a, 2b) despite lacking any training data specifically in this composition regime. These results indicate that the GAP model is capable of predicting the structure of $\mathrm{LiCl}-\mathrm{KCl}$ across all relevant compositions. This performance is a direct result of the use of AL to sample diverse configurations, and introduces the potential to develop ML-IP for binary MS mixtures of arbitrary composition.

Use of the validated GAP models provides insight into the atomistic ordering of $\mathrm{LiCl}-\mathrm{KCl}$ melts across all compositions. As $\mathrm{KCl}$ content decreases in the mixture, the peak in the pair distribution curve near $3.1 \stackrel{\AA}{A}$ also decreases, which can be attributed to the decreased amount of $\mathrm{K}-\mathrm{Cl}$ bond in the melt (Fig. 2a). This also manifests in the reduced structure factor, as the peaks near $4 \AA^{-1}$ and $5 \AA^{-1}$ both increase (Fig. 2b). Unlike $\mathrm{MgCl}_{2}-\mathrm{KCl}$ mixtures, which contain a single network former $(\mathrm{Cl}-\mathrm{Mg}-\mathrm{Cl})$ 
a)

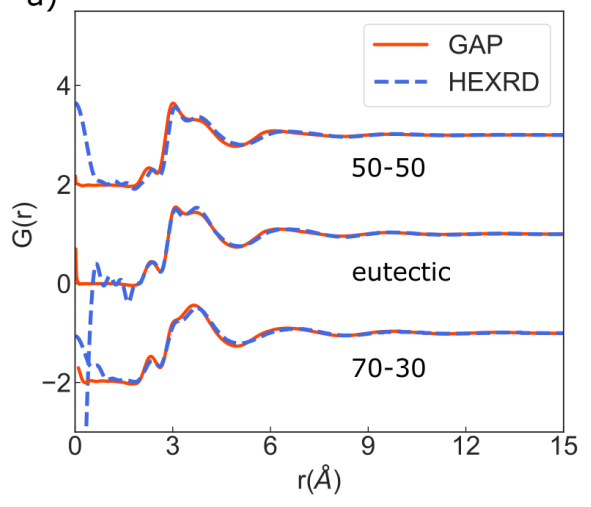

b)

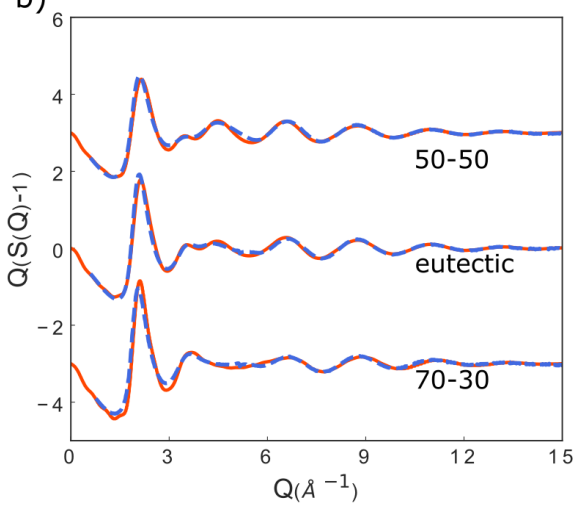

c)

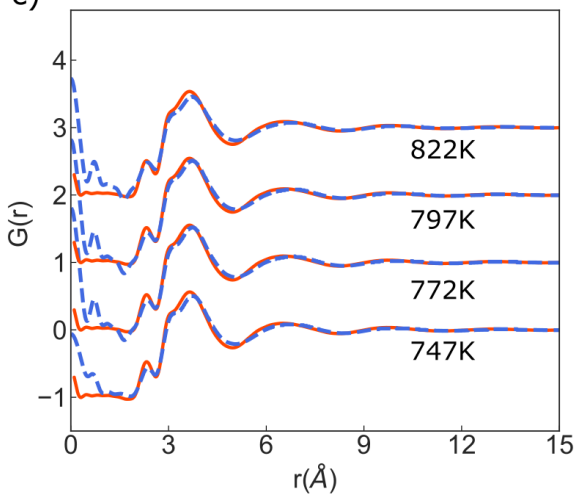

FIG. 2. GAP-predicted structures of molten LiCl-KCl compared to those measured by HEXRD: (a) PDFs and (b) reduced structure factors of $\mathrm{LiCl}-\mathrm{KCl}$ with compositions of $50-50 \mathrm{~mol} \%, 58.5-41.5 \mathrm{~mol} \%$ (eutectic) and 70-30 mol\%, at 757.15K, $645.15 \mathrm{~K}$, and $747.15 \mathrm{~K}$; (c) PDFs of molten $70-30 \mathrm{~mol} \% \mathrm{LiCl}-\mathrm{KCl}$ at $747 \mathrm{~K}, 772 \mathrm{~K}, 797 \mathrm{~K}$, and $822 \mathrm{~K}$.

and a single network breaker $(\mathrm{K}-\mathrm{Cl})$, the components in $\mathrm{LiCl}-\mathrm{KCl}$ are both network breakers [52, 60]. Therefore, we do not expect that the variation of $\mathrm{LiCl}$ and $\mathrm{KCl}$ content will change the mid-range (i.e., on the scale of $1 \mathrm{~nm}$ ) ordering of the melt. To further elucidate the preferential interaction/exclusion of $\mathrm{K} / \mathrm{Li}$ with $\mathrm{Cl}$ at short range (i.e., $\mathrm{r}<5 \AA$ A), we have computed the local/bulk partition coefficient $\left(K_{P}(r)\right)$ as described in supplementary material section $\mathrm{E}[61,62]$. The supplementary material section E equation (2) can be interpreted as $K_{P}(r)>1$ in regions where $\mathrm{K}$ preferentially interact with $\mathrm{Cl}$ and $K_{P}(r)<1$ in regions where Li preferentially interact with $\mathrm{Cl}$. The composition dependence of $K_{P}(r)$ estimated at a fixed temperature of $747 \mathrm{~K}$ is shown in supplementary material section E Fig. 2. All three compositions have a $K_{P}(r)>1$ at short range, showing a preferential interaction of $\mathrm{K}$ with $\mathrm{Cl}$. Further, at short range the maximum values of $K_{P}(r)$ show a trend of 70:30 > Eutectic $>50: 50$, indicating an increase of preferential interaction of $\mathrm{K}$ with respect to $\mathrm{Cl}$ with decreasing content of $\mathrm{KCl}$ in the mixture. Complementary to the information in Fig. 2c, the temperature dependence of $K_{P}(r)$ at the 70:30 composition are visualized in supplementary material section E Fig. 3. It can be observed that temperature does not lead to any significant change in $K_{P}(r)$. We also examined the coordination number of $\mathrm{Li}-\mathrm{Cl}$ and $\mathrm{K}-\mathrm{Cl}$ of $\mathrm{LiCl}-\mathrm{KCl}$ mixtures with different compositions by integrating the partial PDF to the first minimum, and found that the coordination number decreases as the $\mathrm{KCl}$ content increases in the mixture at similar temperatures (i.e., $747 \mathrm{~K}$ to $757 \mathrm{~K}$ ). This might be due to the larger size of $\mathrm{K}$ ions that reduce the number density of the melt. Increased temperature also leads to $\mathrm{Li}-\mathrm{Cl}$ and $\mathrm{K}-\mathrm{Cl}$ coordination numbers decreasing, however, when the change is difficult to distinguish when the temperature variation magnitude is less than 100K. Details of the coordination number changes are listed in Table I.

\begin{tabular}{|c|c|c|c|}
\hline Composition & Temperature $(\mathrm{K})$ & $\mathrm{Li}-\mathrm{Cl}$ & $\mathrm{K}-\mathrm{Cl}$ \\
\hline \multirow{3}{*}{$70-30$} & 747 & 4.42 & 7.28 \\
\cline { 2 - 4 } & 772 & 4.37 & 7.40 \\
\cline { 2 - 4 } & 797 & 4.45 & 7.27 \\
\cline { 2 - 4 } & 822 & 4.42 & 7.37 \\
\hline \multirow{3}{*}{ Eutectic } & 645 & 4.56 & 7.50 \\
\hline 50-50 & 747 & 4.21 & 6.90 \\
\hline
\end{tabular}

TABLE I. Coordination numbers estimated from GAP-MD

Finally, we assessed if the accurate prediction of structure translated to the prediction of thermophysical properties, specifically the thermal conductivity at the eutectic composition. We estimated the thermal conductivity by using the wave method [63]. A detailed discussion on the wave method and calculations are available in supplementary material section F and G. Here we applied GAP MD simulation to predict the thermal conductivity of eutectic $\mathrm{LiCl}-\mathrm{KCl}$ at $645.15 \mathrm{~K}$. The value obtained from GAP MD $(0.659 \mathrm{~W} /(\mathrm{m} . \mathrm{K}))$ is very close to the experimental value [41] $(0.690 \mathrm{~W} /(\mathrm{m} . \mathrm{K}))$, whereas the RIM value is significantly overestimated $(0.856 \mathrm{~W} /(\mathrm{m} . \mathrm{K}))$.

The AL-enabled, and HEXRD-validated, ML-IP workflow described in this letter allows for the rapid characterization of multi-component molten salts of arbitrary compositions and will find broad applicability to other eutectic salt systems. This workflow accelerates exploration processes aimed at identifying the optimal MS composition for target applications by scanning the entire composition map, while simultaneously enabling the real-time deconvolution of complex chemical structures obtained from HEXRD experiments.

This material is based upon work supported by Laboratory Directed Research and Development (LDRD2020-0226, LDRD-CLS-1-630) funding from Argonne National Laboratory, provided by the Director, Office of Science, of the U.S. Department of Energy under Con- 
tract No. DE-AC02-06CH11357. This research was supported in part by the Exascale Computing Project (17SC-20-SC) of the U.S. Department of Energy (DOE), by DOE's Advanced Scientific Research Office (ASCR) under contract DE-AC02-06CH11357 [64]. We gratefully acknowledge the computing resources provided on Bebop; a high-performance computing cluster operated by the Laboratory Computing Resource Center at Argonne National Laboratory. This research used resources of the Argonne Leadership Computing Facility, a DOE Office of Science User Facility supported under Contract DEAC02-06CH11357. HEXRD measurements were made on beamline 6-ID-D at the Advanced Photon Source, a U.S. Department of Energy (DOE) Office of Science User Facility operated for the DOE Office of Science by Argonne National Laboratory under Contract No. DEAC02-06CH11357. Argonne National Laboratory's work was supported by the U.S. Department of Energy, Office of Science, under contract DE-AC02-06CH11357. G. S. would like to thank Prof. Gabor Csányi for constructive feedback on the manuscript and fruitful discussions on GAP model fitting. G.S. would like to Dr. Anand Narayanan Krishnamoorthy for fruitful discussions on local/bulk partition coefficient.

* gsivaraman@anl.gov

[1] J. Dupont, From molten salts to ionic liquids: a "nano" journey, Accounts of Chemical Research 44, 1223 (2011).

[2] H. Kim, D. A. Boysen, J. M. Newhouse, B. L. Spatocco, B. Chung, P. J. Burke, D. J. Bradwell, K. Jiang, A. A. Tomaszowska, K. Wang, et al., Liquid metal batteries: past, present, and future, Chemical Reviews 113, 2075 (2013).

[3] H. Gougar, D. Petti, P. Demkowicz, W. Windes, G. Strydom, J. Kinsey, J. Ortensi, M. Plummer, W. Skerjanc, R. Williamson, et al., The us department of energy's high temperature reactor research and development programprogress as of 2019, Nuclear Engineering and Design 358, 110397 (2020).

[4] J. Guo, N. Hoyt, and M. Williamson, Multielectrode array sensors to enable long-duration corrosion monitoring and control of concentrating solar power systems, Journal of Electroanalytical Chemistry 884, 115064 (2021).

[5] M. Mehos, C. Turchi, J. Vidal, M. Wagner, Z. Ma, C. Ho, W. Kolb, C. Andraka, and A. Kruizenga, Concentrating solar power Gen3 demonstration roadmap, Tech. Rep. (National Renewable Energy Lab, Golden, CO, United States, 2017).

[6] J. Jerden, Molten Salt Thermophysical Properties Database Development: 2019 Update, Tech. Rep. (Argonne National Lab, Argonne, IL, United States, 2019).

[7] J. W. Mcmurray, K. Johnson, C. Agca, B. R. Betzler, D. J. Kropaczek, T. M. Besmann, D. Andersson, and N. Ezell, Roadmap for thermal property measurements of Molten Salt Reactor systems, Tech. Rep. (Oak Ridge National Lab.(ORNL), Oak Ridge, TN (United States), 2021).
[8] S. Jayaraman, A. P. Thompson, and O. A. von Lilienfeld, Molten salt eutectics from atomistic simulations, Physical Review E 84, 030201 (2011).

[9] M. Born and J. E. Mayer, Zur gittertheorie der ionenkristalle, Zeitschrift für Physik 75, 1 (1932).

[10] F. Fumi and M. Tosi, Ionic sizes and born repulsive parameters in the NaCl-type alkali halides- $\mathrm{I}$ : The HugginsMayer and Pauling forms, Journal of Physics and Chemistry of Solids 25, 31 (1964).

[11] P. A. Madden and M. Wilson, 'covalent'effects in 'ionic'systems, Chemical Society Reviews 25, 339 (1996).

[12] M. M. Ghahremanpour, P. J. Van Maaren, and D. Van Der Spoel, The alexandria library, a quantum-chemical database of molecular properties for force field development, Scientific Data 5, 1 (2018).

[13] M. Salanne and P. A. Madden, Polarization effects in ionic solids and melts, Molecular Physics 109, 2299 (2011).

[14] H. Wang, R. S. DeFever, Y. Zhang, F. Wu, S. Roy, V. S. Bryantsev, C. J. Margulis, and E. J. Maginn, Comparison of fixed charge and polarizable models for predicting the structural, thermodynamic, and transport properties of molten alkali chlorides, The Journal of Chemical Physics 153, 214502 (2020).

[15] J. Behler and G. Csányi, Machine learning potentials for extended systems: a perspective, The European Physical Journal B 94, 1 (2021).

[16] V. L. Deringer, A. P. Bartók, N. Bernstein, D. M. Wilkins, M. Ceriotti, and G. Csányi, Gaussian process regression for materials and molecules, Chemical Reviews 121, 10073 (2021).

[17] M. Ceriotti, C. Clementi, and O. Anatole von Lilienfeld, Machine learning meets chemical physics, The Journal of Chemical Physics 154, 160401 (2021).

[18] Y. LeCun, Y. Bengio, and G. Hinton, Deep learning, nature 521, 436 (2015).

[19] A. P. Bartók, M. C. Payne, R. Kondor, and G. Csányi, Gaussian approximation potentials: The accuracy of quantum mechanics, without the electrons, Physical review letters 104, 136403 (2010).

[20] J. Behler and M. Parrinello, Generalized neural-network representation of high-dimensional potential-energy surfaces, Physical review letters 98, 146401 (2007).

[21] I. S. Novikov, K. Gubaev, E. V. Podryabinkin, and A. V. Shapeev, The mlip package: moment tensor potentials with mpi and active learning, Machine Learning: Science and Technology 2, 025002 (2020).

[22] L. Zhang, J. Han, H. Wang, R. Car, and E. Weinan, Deep potential molecular dynamics: a scalable model with the accuracy of quantum mechanics, Physical review letters 120, 143001 (2018).

[23] A. M. Cooper, J. Kästner, A. Urban, and N. Artrith, Efficient training of ann potentials by including atomic forces via taylor expansion and application to water and a transition-metal oxide, npj Computational Materials 6 , $1(2020)$.

[24] A. P. Thompson, L. P. Swiler, C. R. Trott, S. M. Foiles, and G. J. Tucker, Spectral neighbor analysis method for automated generation of quantum-accurate interatomic potentials, Journal of Computational Physics 285, 316 (2015).

[25] S. Batzner, A. Musaelian, L. Sun, M. Geiger, J. P. Mailoa, M. Kornbluth, N. Molinari, T. E. Smidt, and B. Kozinsky, Se (3)-equivariant graph neural networks for 
data-efficient and accurate interatomic potentials, arXiv preprint arXiv:2101.03164 (2021).

[26] F. Musil, M. Veit, A. Goscinski, G. Fraux, M. J. Willatt, M. Stricker, T. Junge, and M. Ceriotti, Efficient implementation of atom-density representations, The Journal of Chemical Physics 154, 114109 (2021).

[27] J. S. Smith, O. Isayev, and A. E. Roitberg, Ani-1: an extensible neural network potential with dft accuracy at force field computational cost, Chemical science 8, 3192 (2017).

[28] K. T. Schütt, H. E. Sauceda, P.-J. Kindermans, A. Tkatchenko, and K.-R. Müller, Schnet-a deep learning architecture for molecules and materials, The Journal of Chemical Physics 148, 241722 (2018).

[29] S. Tovey, A. Narayanan Krishnamoorthy, G. Sivaraman, J. Guo, C. Benmore, A. Heuer, and C. Holm, Dft accurate interatomic potential for molten nacl from machine learning, The Journal of Physical Chemistry C 124, 25760 (2020).

[30] S.-C. Lee, Y. Zhai, Z. Li, N. P. Walter, M. Rose, B. J. Heuser, and Y. Z, Comparative studies of the structural and transport properties of molten salt flinak using the machine-learned neural network and reparametrized classical forcefields, The Journal of Physical Chemistry B 125, 10562 (2021), pMID: 34496565, https://doi.org/10.1021/acs.jpcb.1c05608.

[31] S. T. Lam, Q.-J. Li, R. Ballinger, C. Forsberg, and J. Li, Modeling lif and flibe molten salts with robust neural network interatomic potential, ACS Applied Materials \& Interfaces (2021).

[32] M.-T. Nguyen, R. Rousseau, P. D. Paviet, and V.-A. Glezakou, Actinide molten salts: A machine-learning potential molecular dynamics study, ACS Applied Materials \& Interfaces (2021).

[33] J. Zhang, J. Fuller, and Q. An, Coordination and thermophysical properties of transition metal chlorocomplexes in licl-kcl eutectic, The Journal of Physical Chemistry B 125, 8876 (2021).

[34] K. Liu, Y.-L. Liu, Z.-F. Chai, and W.-Q. Shi, Evaluation of the electroextractions of ce and nd from licl-kcl molten salt using liquid ga electrode, Journal of The Electrochemical Society 164, D169 (2017).

[35] P. D. Myers Jr and D. Y. Goswami, Thermal energy storage using chloride salts and their eutectics, Applied Thermal Engineering 109, 889 (2016).

[36] A. Merwin, M. A. Williamson, J. L. Willit, and D. Chidambaram, Metallic lithium and the reduction of actinide oxides, Journal of the Electrochemical Society 164, H5236 (2017).

[37] A. P. Bartók, R. Kondor, and G. Csányi, On representing chemical environments, Physical Review B 87, 184115 (2013).

[38] V. L. Deringer and G. Csányi, Machine learning based interatomic potential for amorphous carbon, Physical Review B 95, 094203 (2017).

[39] G. Sivaraman, J. Guo, L. Ward, N. Hoyt, M. Williamson, I. Foster, C. Benmore, and N. Jackson, Automated development of molten salt machine learning potentials: Application to licl, The Journal of Physical Chemistry Letters 12, 4278 (2021).

[40] C. G. Staacke, H. H. Heenen, C. Scheurer, G. Csányi, K. Reuter, and J. T. Margraf, On the role of long-range electrostatics in machine-learned interatomic potentials for complex battery materials, ACS Applied Energy Ma- terials 4, 12562 (2021).

[41] G. J. Janz, Molten salts handbook (Elsevier, 2013).

[42] G. Sivaraman, A. N. Krishnamoorthy, M. Baur, C. Holm, M. Stan, G. Csányi, C. Benmore, and Á. VázquezMayagoitia, Machine-learned interatomic potentials by active learning: amorphous and liquid hafnium dioxide, npj Computational Materials 6, 1 (2020).

[43] G. Sivaraman, L. Gallington, A. N. Krishnamoorthy, M. Stan, G. Csányi, Á. Vázquez-Mayagoitia, and C. J. Benmore, Experimentally driven automated machinelearned interatomic potential for a refractory oxide, Physical Review Letters 126, 156002 (2021).

[44] J. Sun, A. Ruzsinszky, and J. P. Perdew, Strongly constrained and appropriately normed semilocal density functional, Physical review letters 115, 036402 (2015).

[45] J. Sun, R. C. Remsing, Y. Zhang, Z. Sun, A. Ruzsinszky, H. Peng, Z. Yang, A. Paul, U. Waghmare, X. Wu, et al., Accurate first-principles structures and energies of diversely bonded systems from an efficient density functional, Nature chemistry 8, 831 (2016).

[46] L. Ward, G. Sivaraman, J. G. Pauloski, Y. Babuji, R. Chard, N. Dandu, P. C. Redfern, R. S. Assary, K. Chard, L. A. Curtiss, et al., Colmena: Scalable machine-learning-based steering of ensemble simulations for high performance computing, arXiv preprint arXiv:2110.02827 (2021).

[47] A. Laio and M. Parrinello, Escaping free-energy minima, Proceedings of the National Academy of Sciences 99, 12562 (2002).

[48] N. Bernstein, G. Csányi, and V. L. Deringer, De novo exploration and self-guided learning of potential-energy surfaces, npj Computational Materials 5, 1 (2019).

[49] L. Bonati and M. Parrinello, Silicon liquid structure and crystal nucleation from ab initio deep metadynamics, Physical review letters 121, 265701 (2018).

[50] M. Bonomi, Promoting transparency and reproducibility in enhanced molecular simulations, Nature methods 16, 670 (2019).

[51] A. Barducci, G. Bussi, and M. Parrinello, Well-tempered metadynamics: a smoothly converging and tunable freeenergy method, Physical review letters 100, 020603 (2008).

[52] F. Wu, S. Sharma, S. Roy, P. Halstenberg, L. C. Gallington, S. M. Mahurin, S. Dai, V. S. Bryantsev, A. S. Ivanov, and C. J. Margulis, Temperature dependence of short and intermediate range order in molten $\mathrm{mgcl} 2$ and its mixture with kcl, The Journal of Physical Chemistry B 124, 2892 (2020).

[53] S. Plimpton, Fast parallel algorithms for short-range molecular dynamics, Journal of computational physics 117, 1 (1995).

[54] G. Csányi, S. Winfield, J. R. Kermode, A. De Vita, A. Comisso, N. Bernstein, and M. C. Payne, Expressive programming for computational physics in fortran 95+, IoP Comput. Phys. Newsletter, Spring 2007 (2007).

[55] S. Nosé, A unified formulation of the constant temperature molecular dynamics methods, The Journal of chemical physics 81, 511 (1984).

[56] W. G. Hoover, Canonical dynamics: Equilibrium phasespace distributions, Physical review A 31, 1695 (1985).

[57] M. Parrinello and A. Rahman, Polymorphic transitions in single crystals: A new molecular dynamics method, Journal of Applied physics 52, 7182 (1981). 
[58] G. J. Martyna, D. J. Tobias, and M. L. Klein, Constant pressure molecular dynamics algorithms, The Journal of chemical physics 101, 4177 (1994).

[59] W. Shinoda, M. Shiga, and M. Mikami, Rapid estimation of elastic constants by molecular dynamics simulation under constant stress, Physical Review B 69, 134103 (2004).

[60] M. Wilson and P. Madden, "prepeaks"and "first sharp diffraction peaks" in computer simulations of strong and fragile ionic liquids, Physical review letters 72, 3033 (1994).

[61] F. Rodriguez-Ropero and N. F. van der Vegt, Direct osmolyte-macromolecule interactions confer entropic stability to folded states, The Journal of Physical Chemistry
B 118, 7327 (2014).

[62] A. N. Krishnamoorthy, J. Zeman, C. Holm, and J. Smiatek, Preferential solvation and ion association properties in aqueous dimethyl sulfoxide solutions, Physical Chemistry Chemical Physics 18, 31312 (2016).

[63] B. Cheng and D. Frenkel, Computing the heat conductivity of fluids from density fluctuations, Physical Review Letters 125, 130602 (2020).

[64] F. J. Alexander, J. Ang, J. A. Bilbrey, J. Balewski, T. Casey, R. Chard, J. Choi, S. Choudhury, B. Debusschere, A. M. DeGennaro, et al., Co-design center for exascale machine learning technologies (exalearn), The International Journal of High Performance Computing Applications , 10943420211029302 (2020). 


\section{Supplemental materials to the manuscript "A Composition-Transferable Machine Learning Potential for LiCl-KCl Molten Salts Validated by HEXRD"}

\section{A. GAP TRAINING DATASET AND HYPERPARAMETER}

\begin{tabular}{|c|c|}
\hline System & Number of samples \\
\hline Melt & 928 \\
\hline Metadynamics & 196 \\
\hline Isolated atoms & 3 \\
\hline Total & $\mathbf{1 1 2 7}$ \\
\hline
\end{tabular}

TABLE I: GAP training database

\begin{tabular}{|c|c|c|}
\hline Parameter Name & Two-body & SOAP \\
\hline Cut off $(\AA)$ & 5.92 & 5.92 \\
\hline Sparse method & Uniform & CUR \\
\hline Sparse points & 65 & 1200 \\
\hline Delta $(\mathrm{eV})$ & 2.74 & 0.78 \\
\hline$\left(l_{\max }, n_{\max }\right)$ & - & $(4,8)$ \\
\hline
\end{tabular}

TABLE II: GAP model hyperparameter

The GAP model (Table I, II) validated on 120 independent test samples showed an root mean square error in energy and force of $5 \mathrm{meV} /$ atom and $0.12( \pm 0.04) \mathrm{eV} / \stackrel{\AA}{A}$, respectively.

\section{B. DENSITY FUNCTIONAL THEORY}

DFT single point calculations are performed using the Vienna ab initio simulation package [1]. The SCAN exchangecorrelation functional and projector-augmented wave method are employed [2, 3]. A large plane wave cutoff of 700 $\mathrm{eV}$ with an electronic convergence criterion of $10^{-7} \mathrm{eV}$ is used. $\mathrm{A} \Gamma$-centered $1 \times 1 \times 1 \mathrm{k}$-mesh is used for reciprocal sampling.

\section{METADYNAMICS SAMPLING}

The metadynamics calculations are performed using the plumed 2 packages [4]. A coordination number collective variable is chosen. For any two arbitrary chemical species groups A and B, they are estimated using the following switch function:

$$
C_{A B}=\sum_{i \in A, j \in B} \frac{1-\left(\frac{r_{i j}}{r_{0}}\right)^{6}}{1-\left(\frac{r_{i j}}{r_{0}}\right)^{12}}
$$

with $\mathrm{r}_{i j}$ being the distances between the atom-atom pairs from the species groups.

A system size of 64 size with 16 anion-cation pairs each for $\mathrm{Li}-\mathrm{Cl}$ and $\mathrm{K}-\mathrm{Cl}$ is used for sampling. The $\mathrm{r}_{0}$ was parametrized from the first minima of the partial pair distributions functions. For Li-Cl and $\mathrm{K}-\mathrm{Cl}$ the $\mathrm{r}_{0}$ of $3.35 \stackrel{A}{\circ}$ and $4.25 \AA$ respectively are found to be optimal. The metadynamics used an initial Gaussian height of $40 \mathrm{~kJ} / \mathrm{mol}$. The Gaussian widths of $1.45,2.58$ are used for $\mathrm{C}_{L i C l}$, and $\mathrm{C}_{K C l}$ respectively. A Gaussian was deposited every 250 fs with a bias factor equal to 50 . 


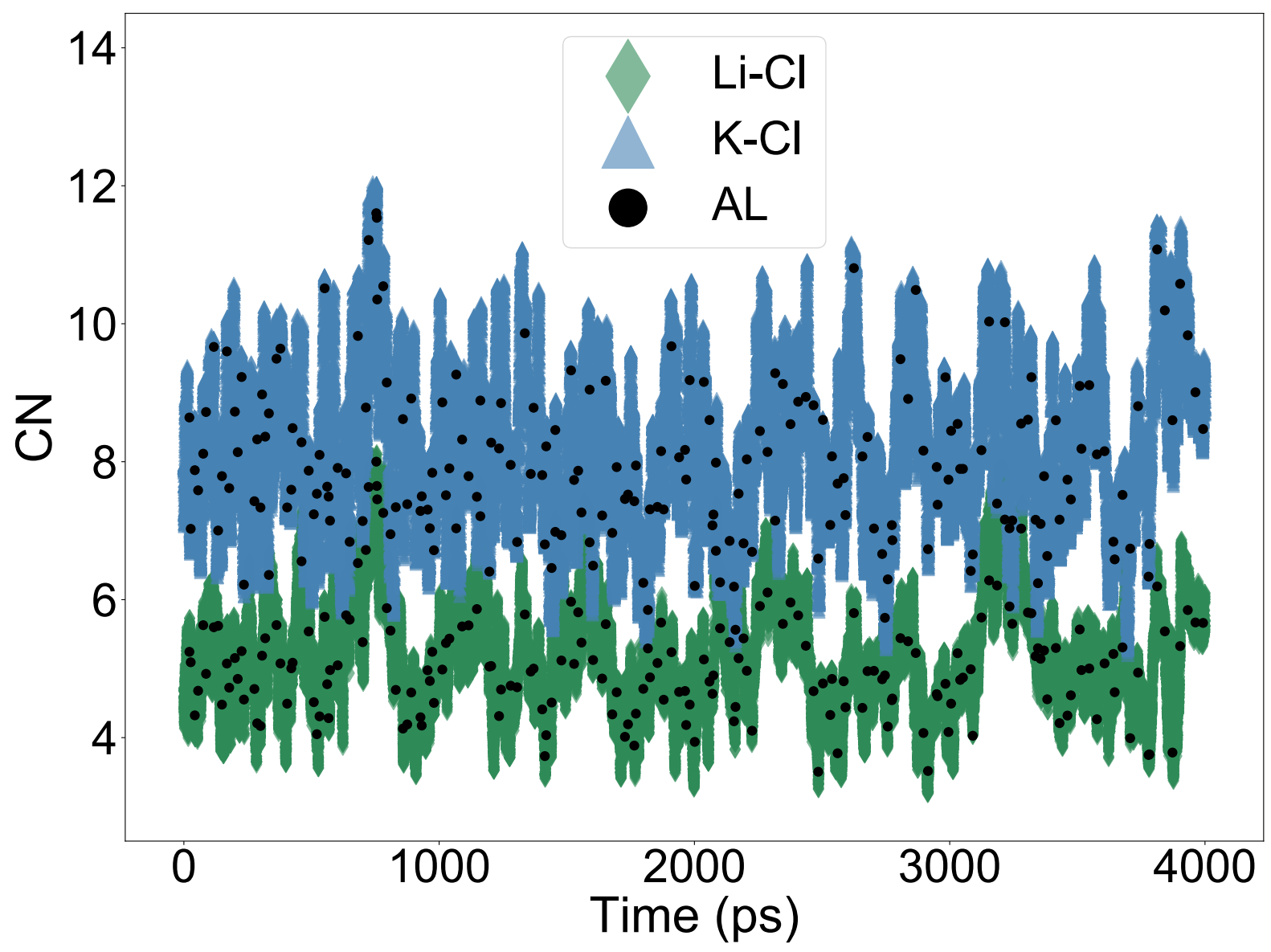

FIG. 1: Evolution of the anion-cation pair collective variable for the 50:50 composition over 4 ns of well-tempered metadynamics performed using the intermediate GAP-ML model. The configurations selected by active learning for single point DFT are shown as black dots. The y-axis shows coordination number $(\mathrm{CN})$ averaged over 16 anion-cation pair used in the CV definition.

\section{GAP-MD SETTINGS AND RESULTS}

\begin{tabular}{|c|c|c|c|c|}
\hline Composition (LiCl:KCl) & Number of atoms & Temperature $(\mathrm{K})$ & Initial density $\left(\mathrm{g} \cdot \mathrm{cm}^{-3}\right)$ & Density from GAP-MD $\left(\mathrm{g} . \mathrm{cm}^{-3}\right)$ \\
\hline \multirow{3}{*}{$70-30$} & \multirow{3}{*}{1120} & 747.15 & 1.602 & $1.696( \pm 0.012)$ \\
\cline { 3 - 5 } & & 772.15 & 1.602 & $1.692( \pm 0.012)$ \\
\cline { 3 - 5 } & & 797.15 & 1.602 & $1.690( \pm 0.012)$ \\
\cline { 3 - 5 } & 1040 & 822.15 & 1.602 & $1.685( \pm 0.012)$ \\
\hline Eutectic & 1024 & 757.15 & 1.622 & $1.558( \pm 0.015)$ \\
\hline $50-50$ & 1120 & 900 & 1.584 & $1.555( \pm 0.015)$ \\
\hline $30-70$ & 960 & 800 & 1.583 & $1.764( \pm 0.012)$ \\
\hline $80-20$ & & 1.578 & $0.012)$ \\
\hline
\end{tabular}

TABLE III: GAP MD simulation set up. A 50-50 composition of $\mathrm{LiCl}: \mathrm{KCl}$ with 1024 atom system is equal to 256 anion-cation pairs for each salt. The initial density and targer temperature used for MD simulation. The density computed from the last $1 \mathrm{~ns}$ of GAP-MD based NPT simulation along with standard deviations (parenthesis) are reported in the last column. The additional compositions $80-20$, and $30-70$ are computed to show case the capability of the GAP model to predict outside of experiment conditions. 


\section{E. LOCAL/BULK PARTITION COEFFICIENT}

The local/bulk partition coefficient $[5,6]$ is defined as

$$
K_{P}=\frac{\left(\left\langle n_{\alpha}(r)\right\rangle /\left\langle n_{\beta}(r)\right\rangle\right)^{l o c a l}}{\left(n_{\alpha}^{\text {tot }} / n_{\beta}^{\text {tot }}\right)^{\text {bulk }}}
$$

where $\left\langle n_{X}(r)\right\rangle$ indicates the cumulative number distribution function of $\mathrm{X}(\mathrm{X}=\alpha$ for $\mathrm{K}$ around $\mathrm{Cl}, \mathrm{X}=\beta$ for $\mathrm{Li}$ around $\mathrm{Cl}$ ) at a distance $\mathrm{r}$ and $n_{X}^{\text {tot }}$ indicated the total number of $\mathrm{X}$ in the simulation box. $K_{P}>1$ indicated a preferential interaction of $\mathrm{K}$ with respect to $\mathrm{Cl}$.

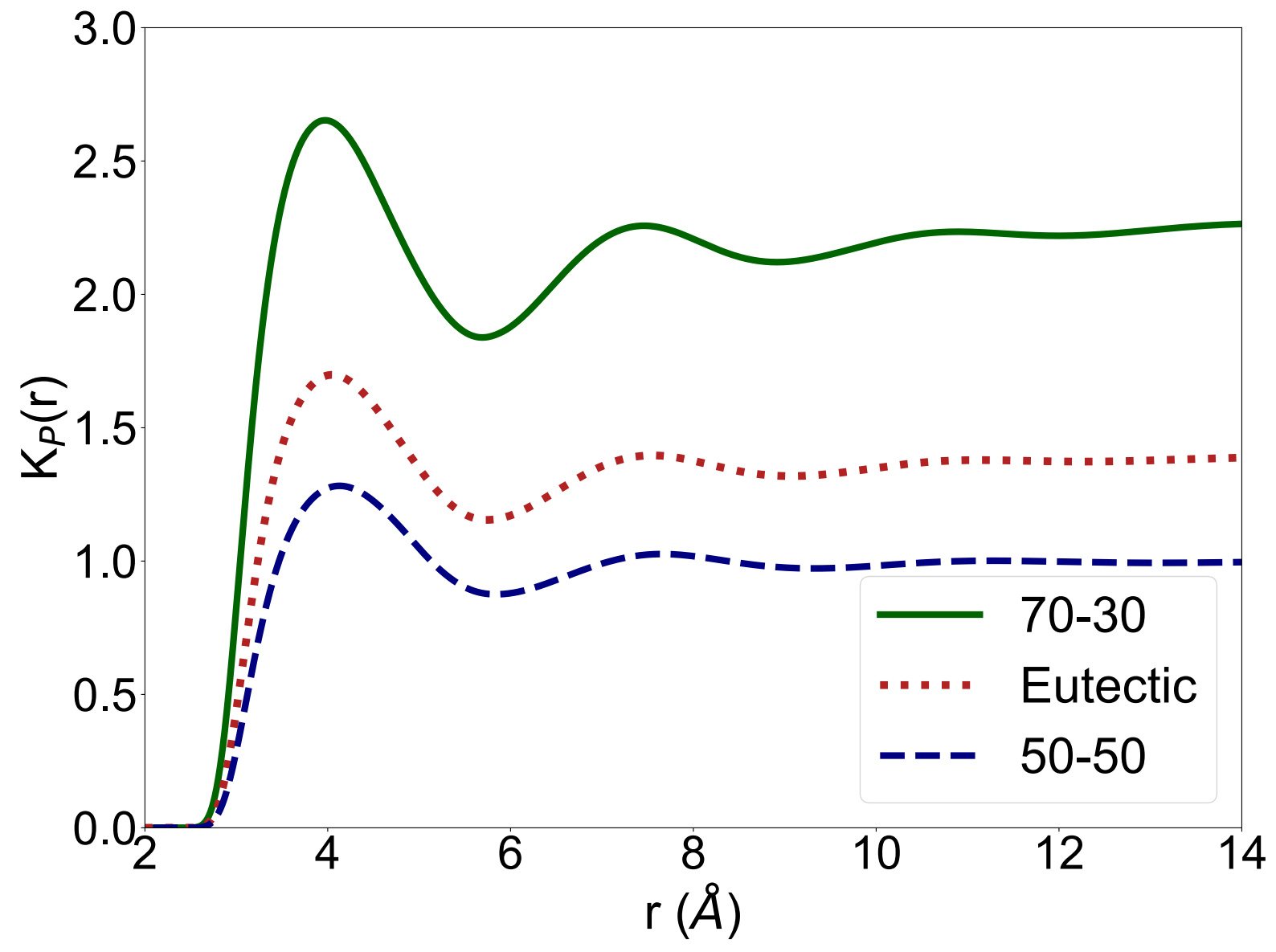

FIG. 2: Local/bulk partition coefficient computed at $747 \mathrm{~K}$ as function of LiCl: $\mathrm{KCl}$ composition. 


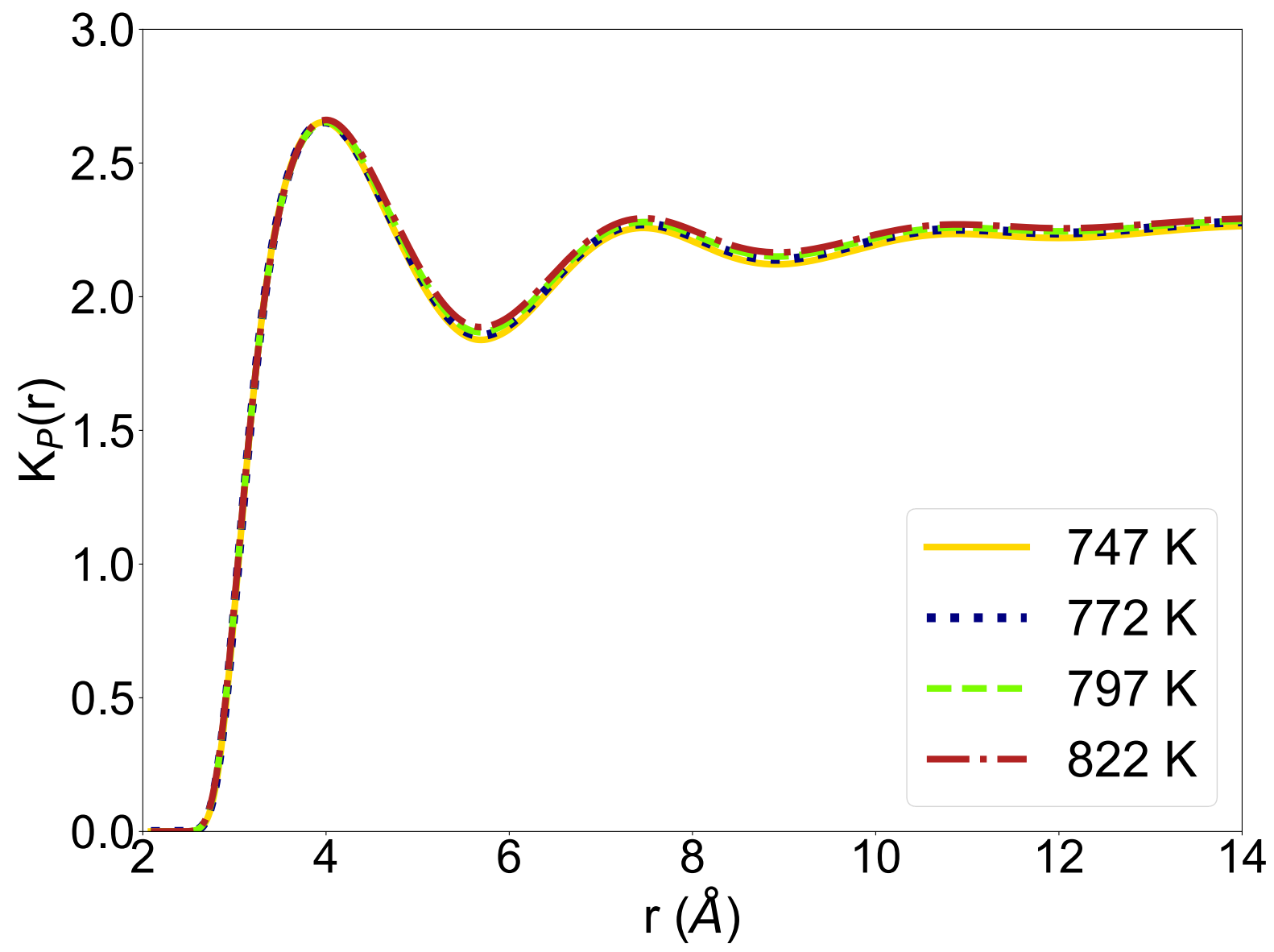

FIG. 3: Local/bulk partition coefficient computed for LiCl:KCl composition of 70:30 as function of temperature.

\section{F. THERMAL CONDUCTIVITY}

The thermal conductivity estimated from virial stress based heat flux implemented with LAMMPS is not valid beyond pair interaction models $[7,8]$. Hence they cannot be applied to many-body ML-IP such GAP model. To bypass this problem we will estimate the thermal diffusivity of eutectic liquid using the thermally driven liquid density fluctuation at hydrodynamic limit (i.e. as system size goes to infinity, $k \rightarrow 0$ ) $[9,10]$. The most relevant details of the approach are reproduced below. The density field of the liquid in bulk limit $(\rho(r, t))$ is computed from equilibrium MD simulation. For a periodic system with dimension of $\left\{L_{x}, L_{y}, L_{z}\right\}$, the Fourier transform of the density field $\rho(r, t)$ in a bulk liquid is defined as:

$$
\widetilde{\rho}(k, t)=\frac{1}{V} \sum_{i=1}^{N} \exp \left(-i k . r_{i}(t)\right),
$$

where the reciprocal lattice vector, $k=\left\{\frac{2 \pi n_{x}}{L_{x}}, \frac{2 \pi n_{y}}{L_{y}}, \frac{2 \pi n_{z}}{L_{z}}\right\}$. The power spectrum of the density field fluctuation with frequency $\omega$ is defined as:

$$
S(k, \omega)=\frac{1}{T_{0}} \int_{0}^{T_{0}}\langle\widetilde{\rho}(k, 0) \widetilde{\rho}(k, t)\rangle \exp (-i \omega t) d t
$$

The $S(k, \omega)$ derived from the MD simulation can be fitted to power spectrum equation derived from hydrodynamic theory: 


$$
S(k, \omega)=\frac{S(k)}{2 \pi}\left[\left(\frac{\gamma-1}{\gamma}\right) \frac{2 D_{T} k^{2}}{\omega^{2}+\left(D_{T} k^{2}\right)^{2}}+\frac{1}{\gamma}\left(\frac{\Gamma k^{2}}{\left(\omega+c_{s} k\right)^{2}+\left(\Gamma k^{2}\right)^{2}}+\frac{\Gamma k^{2}}{\left(\omega-c_{s} k\right)^{2}+\left(\Gamma k^{2}\right)^{2}}\right)\right]
$$

where, $\gamma=c_{P} / c_{V}$ is the ratio of particle specific heat capacities (defined below), $D_{T}$ is the thermal diffusivity, $c_{s}$ is the adiabatic speed of sound, $\mathrm{b}$ is the kinematic longitudinal viscosity, and the sound attenuation constant $\Gamma=(\gamma-1) D_{T} / 2+b / 2$. The first term in eqn. 5 is related to thermal transport and the last two terms corresponds to sound wave travelling in opposite direction. Further details on the methodology along with the code is discussed in detail elsewhere [10].

Now we will briefly discuss the thermodyanmic relation relvant to quantities that arise in Eqn. 5. These quantities are estimated from numeric partial deviates with respect to state variables as defined below:

$$
\begin{gathered}
c_{V}=\frac{1}{N}\left(\frac{\partial E}{\partial T}\right)_{V} \\
c_{P}-c_{V}=-\frac{T}{N}\left(\frac{\partial P}{\partial T}\right)_{V}^{2}\left(\frac{\partial P}{\partial V}\right)_{T}^{-1} \\
c_{s}^{2}=-\frac{V^{2} \gamma}{N m}\left(\frac{\partial P}{\partial V}\right)_{T}
\end{gathered}
$$

As described in Ref. [10], $D_{T}$ and b will be treated as the fit parameters. Once the $D_{T}$ is obtained by a linear fit, the thermal conductivity $(\lambda)$ can be computed using the following relation:

$$
\lambda=\rho_{0} c_{P} D_{T}
$$

where $\rho_{0}$ is the liquid density.

\section{G. THERMAL CONDUCTIVITY FROM GAP-MD}

The GAP MD simulation was performed for Eutectic system at $645.15 \mathrm{~K}$ with a elongated system [10]. In order to avoid finite size effect a very large $L_{z} \approx 1000 \AA$ is chosen which translates to a system size of 8,320 atoms $(1.558$ g. $\left.\mathrm{cm}^{-3}\right)$. For benchmark purposes we also set up a simulation with RIM model with same number of atoms and elongation $\left(1.512 \mathrm{~g} . \mathrm{cm}^{-3}\right)$. The simulations are performed with in NVT ensemble with a global stochastic velocity rescaling thermostat [11]. A time step of $0.5 \mathrm{fs}$ is used and the simulations are performed for 500 ps. The results are reported in Table IV.

\begin{tabular}{|c|c|c|c|c|c|}
\hline Method & $c_{v}(\mathrm{~J} /(\mathrm{K} . \mathrm{mol}))$ & $\gamma$ & $c_{s}(\AA / \mathrm{ps})$ & $D_{T}\left(\AA^{2} / \mathrm{ps}\right)$ & $\lambda(\mathrm{W} /(\mathrm{m} . \mathrm{K}))$ \\
\hline GAP & 34.1 & 1.21 & 0.434 & $27.5(2)$ & 0.659 \\
\hline RIM & 29.8 & 1.20 & 0.355 & $43.9(2)$ & 0.856 \\
\hline Experiment [12] & - & - & - & - & 0.690 \\
\hline
\end{tabular}

TABLE IV: Fit parameters for eqn. 5 and the estimated thermal conductivity. Linear fit $D_{T}(k)$ is performed as function of $\mathrm{k}$ for all the points where statistical error up to $10^{-2}$ (paranthesis) and extrapolated to $\mathrm{k}=0$ as explained in Ref [10].

[1] G. Kresse and J. Furthmüller, Physical review B 54, 11169 (1996). 
[2] J. Sun, A. Ruzsinszky, and J. P. Perdew, Physical review letters 115, 036402 (2015).

[3] P. E. Blöchl, Physical review B 50, 17953 (1994).

[4] G. A. Tribello, M. Bonomi, D. Branduardi, C. Camilloni, and G. Bussi, Computer Physics Communications 185, 604 (2014).

[5] F. Rodriguez-Ropero and N. F. van der Vegt, The Journal of Physical Chemistry B 118, 7327 (2014).

[6] A. N. Krishnamoorthy, J. Zeman, C. Holm, and J. Smiatek, Physical Chemistry Chemical Physics 18, 31312 (2016).

[7] P. Boone, H. Babaei, and C. E. Wilmer, Journal of chemical theory and computation 15, 5579 (2019).

[8] S. Plimpton, Journal of computational physics 117, 1 (1995).

[9] R. D. Mountain, Reviews of Modern Physics 38, 205 (1966).

[10] B. Cheng and D. Frenkel, Physical Review Letters 125, 130602 (2020).

[11] G. Bussi, D. Donadio, and M. Parrinello, The Journal of chemical physics 126, 014101 (2007).

[12] G. J. Janz, Molten salts handbook (Elsevier, 2013). 